\title{
Modeling non-premixed combustion using tabulated kinetics and different flame structure assumptions
}

\author{
Author, co-author list (Do NOT enter this information. It will be pulled from participant tab in \\ MyTechZone) \\ Affiliation (Do NOT enter this information. It will be pulled from participant tab in MyTechZone)
}

Copyright (c) 2017 SAE International

\begin{abstract}
Nowadays, detailed kinetics is necessary for a proper estimation of both flame structure and pollutant formation in compression ignition engines. However, large mechanisms and the need to include turbulence/chemistry interaction introduce significant computational overheads. For this reason, tabulated kinetics is employed as a possible solution to reduce the CPU time even if table discretization is generally limited by memory occupation. In this work the authors applied tabulated homogeneous reactors (HR) in combination with different turbulent-chemistry interaction approaches to model non-premixed turbulent combustion. The proposed methodologies represent good compromises between accuracy, required memory and computational time. The experimental validation was carried out by considering both constant-volume vessel and Diesel engine experiments. First, the ECN Spray A configuration was simulated at different operating conditions and results from different flame structures are compared with experimental data of ignition delay, flame lift-off, heat release rates, radicals and soot distributions. Afterwards, engine simulations were carried out and computed data are validated by cylinder pressure and heat release rate profiles.
\end{abstract}

\section{Introduction}

High power-to-weight ratio and thermal efficiencies close to $45 \%$ have made compression-ignition engines among the most widely used prime movers for many industrial and road transportation applications. However, recent changes in emission legislations and the need to continuously reduce the fuel consumption require further improvements to the combustion system. In particular, an optimal combination of combustion chamber design, compression ratio and injection strategy has the potential to increase thermal efficiency and, at the same time, ensure acceptable levels of pollutant emissions $[1,2]$.
To speed-up the engine development process and identify the most promising configurations in the shortest amount of time, multi-dimensional numerical models are widely employed to predict Diesel combustion, although such topic represents a very challenging task for computational fluid dynamics due to the need to accurately describe the evolution of a turbulent, compressible, reacting and multiphase flow [3, 4]. Since not only engine performances but emissions as well need to be estimated with a good level of accuracy must be estimated with a good level of accuracy, combustion models have to incorporate detailed chemistry and also the effects of its interaction with turbulence $[5,6,7,8]$. Different combustion approaches are available handling chemical kinetics in two different ways: direct integration and tabulation. In the first case, reactiondiffusion equations are solved on-line during the simulation for any species included in the chemical mechanism. Computation of reaction rates requires the use of ODE stiff solvers since chemical time-scales are smaller than the flow ones, with a consequent increase of the required computational time. Despite the use of parallell computing and the enhancemens of processor performances, the use of very detailed schemes is still prohibitive and maximum number of species which can be employed for practical simulations is generally less than 100 . This aspect represents an important limitation related to the capability to predict the main soot precursor species or ignition delays under advanced combustion modes where high levels of charge dilution are generally used $[9,10]$. To reduce the computational time, different techniques were developed in the past: among them we can mention on-line tabulation or cell clustering: when applied in combination with the well-mixed model they offer acceptable speed-up factors in case relatively small mechanisms (up to 50 species) are employed $[11,12,13,14]$. Inclusion of turbulence-chemistry interaction adds significant overheads related to the need to consider the effects of sub-grid mixing either by integrating a 
presumed PDF or solving additional equations for the different expected flow realizations $[5,15]$. This explains the reason why the most widely used approaches are the wellmixed and the Representative Interactive Flamelet (RIF) ones. The first one accounts for local flow conditions but cannot include turbulence-chemistry interaction, the second one solves reaction-diffusion equations in the mixture fraction $(Z)$ space having a limited number of nodes and then computes the chemical composition in each cell by presuming a $\beta$-PDF distribution for the chemical species which is function of $Z$ and its variance $\widetilde{Z^{\prime \prime 2}}$. When a single flamelet is used, flame stabilization cannot be predicted and extesion to multiple flamelets is computationally demanding. More sophisticated approaches exist, like Conditional Moment Closure or Transported PDF, but their application so far is still limited to advanced research studies.

The need to account properly for turbulence-chemistry interaction and to achieve results within an acceptable amount of time suggests the use of tabulated kinetics: chemical species reaction rates are stored in a table according to a specified mechanism and flame structure; then they are retrieved as function of the state of the system. Different approaches were presented in the past: the first ones used a rigorous mathematical time-scale analysis to identify the relevant species in the mechanism and use them as independent variables for the tabulation [16]. Afterwards, the attention was mainly focused on the capability to reproduce both auto-ignition and diffusive flame structure and for this reason tabulations based on auto-igniting homogeneous reactors $[17,18]$ or diffusive flamelets $[19,20,21]$ were presented and successfully applied to model Diesel combustion. Approximated diffusion flamelets (ADF) represents an interesting compromise between these approaches since they tabulate diffusion flamelets computed with source terms taken from tables generated using homogeneous reactor calculations [22, 23]. To be used in engine simulations, tabulated kinetics must fulfill the following prerequisites:

1. Acceptable table size to avoid excessive memory occupation during the simulation;

2. Consistency of the results with the ones performed using direct integration and the same flame structure assumption;

3. Minimum number of transport equations to be solved in the CFD domain.

Objective of this work is the development of combustion models based on tabulated homogeneous reactors (HR). Different flame structures are considered: well-mixed, presumed PDF and diffusive flamelets. For all of them, related transport equations are solved on-line with reaction rates retrieved from the table. This makes possible to minimize the size of the table and to account in different ways for turbulence-chemistry interaction. Tabulated quantities are stored as function of state variables of the system (temperature, mixture fraction, pressure) and the combustion progress variable. The table includes data of progress variable reaction rate and chemical composition which is represented by a virtual set of seven species preserving the most important thermodynamic properties of the full set used in the mechanism. It is also possible to store mass fractions of additional species which can be employed in sub-models for the computation of pollutant emissions ( soot and $\mathrm{NO}_{x}$ ).

The proposed approach for combustion modeling based on tabulated kinetics was implemented in the Lib-ICE code which is based on the OpenFOAM $®$ technology $[24,25]$. Experiments carried out in the context of the Engine Combustion Network (ECN) using n-dodecane as fuel at constant-volume conditions were used for a complete assessment of the proposed combustion models. Computed results are compared with experimental data but also with the ones available from the same models employing directintegration. The comparison was performed in terms of vessel pressure, heat release rate, ignition delay time, flame lift-off and soot distribution. Afterwards, preliminary results of combustion simulations in a medium-duty Diesel engine are reported.

\section{Computational Model}

This section describes the way tabulated kinetics is handled and how it is incorporated in three different combustion models: well-mixed, presumed PDF and representative interactive flamelets. The following acronyms will be used to easily identify them:

- TWM : tabulated well-mixed model;

- TPPDF : tabulated presumed PDF model;

- TRIF : tabulated representative interactive flamelet.

Results of approaches based on direct integration will be also presented in this paper for model assessment and they will be named as:

- DIWM : well mixed model;

- DIRIF : representative interactive flamelet model.

\section{Chemistry table}

Figure 1 summarizes the way chemistry is tabulated in the proposed approach. The user specifies a chemical mechanism and a range of initial conditions for homogeneous constant pressure reactor calculations in terms of:

- Mixture fraction $Z$;

- Ambient pressure $p$;

- Initial reactor temperature $T_{u}$;

- Residual gas fraction $E G R$;

On the basis of such quantities, initial chemical composition is computed and reactor calculation is started. $T_{u}$ can be initialized in two ways: 
1. Temperature is independent from $\mathrm{Z}$;

2. Reactor temperature varies from values specified at $Z$ $=0$ and $\mathrm{Z}=1$ including, eventually, fuel evaporation:

$$
\begin{gathered}
h(Z)=(1-Z) \cdot h\left(T_{Z=0}\right)+Z \cdot h\left(T_{Z=1}\right)-Z \cdot h_{l}\left(T_{Z=1}\right) \\
T_{u}(Z)=T(h(Z)) ;
\end{gathered}
$$

where $h_{l}$ is the fuel heat of vapourization.

Both approaches are equivalent and provides the same results, since in both cases all possible expected thermochemical states of the system are covered. Option 2 is probably more suitable for table discretization in diffusion combustion problems because the user provides well known values of expected oxidizer temperatures $\left(T_{Z=0}\right)$, fuel temperature and its heat of evaporation.

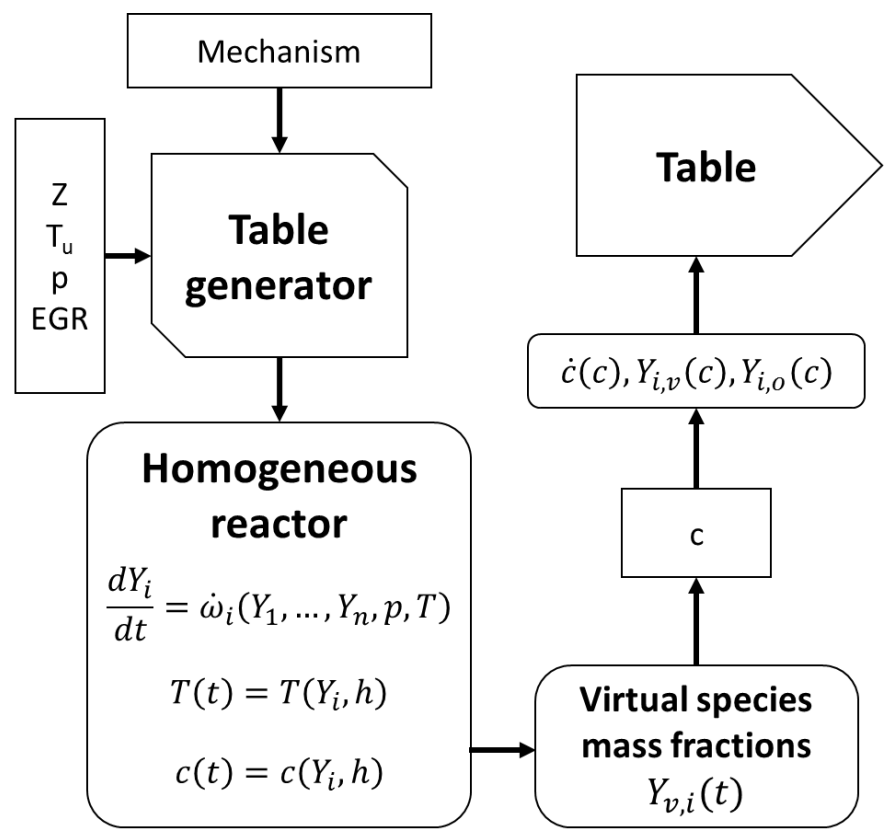

Figure 1: Generation of the chemistry table based on the homogeneous reactor assumption.

For any specified condition, chemical species equations are solved:

$$
\frac{d Y_{i}}{d t}=\dot{\omega}_{i}\left(T, p, Y_{1}, \ldots, Y_{n}\right)
$$

with reactor temperature $T$ computed directly from the initial enthalpy value. After every time step two different operations are performed: evaluation of the progress variable $C$ and computation of the chemical composition by means of the virtual species approach. Definition of the combustion progress variable is not straightforward and it was widely discussed in past works [19, 26, 20]. This aspect is even more complex in case of large hydrocarbon fuel auto-ignition since it might take place either in one or two stages. In this work, the definition proposed by Lehtiniemi et al. [20] was adopted, with $C$ being equal to the heat released by combustion, computed as the difference between the current and the initial value of the reactor formation enthalpy, also known as $h_{298}$ :

$$
C=\sum_{i=1}^{N s} h_{298, i} \cdot Y_{i}(t)-\sum_{i=1}^{N s} h_{298, i} \cdot Y_{i}(0)
$$

where $N_{s}$ is the total number of chemical species used by the specified mechanism. The adopted definition for $C$ uniquely characterizes each point in the thermochemical state space and is appropriate for a transport equation. At the end of each reactor calculation, progress variable reaction rates and chemical composition are stored as function of the discrete values of the normalized progress variable $c$, specified by the user:

$$
c=\frac{C-C_{\min }}{C_{\max }-C_{\min }}
$$

where $C_{\min }$ and $C_{\max }$ are minimum and maximum values of the progress variable which are found at initial and after auto-ignition conditions. $C_{\min }$ and $C_{\max }$ are stored in the table as function of $Z, T_{u}, p$. From the values of times at which the specified $c_{i}$ values were found, the progress variable reaction rate is computed with the forward differencing scheme as follows:

$$
\dot{c}_{i}=\frac{c_{i+1}-c_{i}}{t_{i+1}-t_{i}}
$$

$\dot{c}$ is then multiplied by the term $C_{\max }-C_{\min }$ in order to have the proper source term $\dot{C}$ in the progress variable transport equation. For any tabulated value of the progress variable $c$, the chemical composition is also stored. However, to avoid the storage of the entire set of species and keep an acceptable size of the table, only seven ones named virtual species are tabulated and their mass fractions are computed in order to preserve the main thermochemical properties of the full set used in the detailed mechanism. Virtual species used in this work are $\mathrm{N}_{2}, \mathrm{O}_{2}$, fuel, $\mathrm{CO}_{2}$, $\mathrm{CO}, \mathrm{H}_{2} \mathrm{O}, \mathrm{H}_{2}$ and their composition is computed for any $c$ value as follows:

$$
\begin{aligned}
& \sigma_{H}=\sum_{i=1}^{N_{s}} N_{H, i} \cdot x_{i}=\sum_{k=1}^{N_{v}} N_{H, k} \cdot x_{v, i} \\
& \sigma_{C}=\sum_{i=1}^{N_{s}} N_{C, i} \cdot x_{i}=\sum_{k=1}^{N_{v}} N_{C, k} \cdot x_{v, i} \\
& \sigma_{O}=\sum_{i=1}^{N_{s}} N_{O, i} \cdot x_{i}=\sum_{k=1}^{N_{v}} N_{C, k} \cdot x_{v, i} \\
& \sigma_{N}=\sum_{i=1}^{N_{s}} N_{N, i} \cdot x_{i}=\sum_{k=1}^{N_{v}} N_{N, k} \cdot x_{v, i} \\
& h=\sum_{i=1}^{N_{s}} Y_{i} \cdot h_{i}(T)=\sum_{k=1}^{N_{v}} Y_{i, v} \cdot h_{i}(T)
\end{aligned}
$$




$$
\begin{gathered}
c_{p}=\sum_{i=1}^{N_{s}} Y_{i} \cdot c_{p, i}(T)=\sum_{k=1}^{N_{v}} Y_{i, v} \cdot c_{p, k}(T) \\
\sum_{k=1}^{N_{v}} Y_{i, v}=1.0
\end{gathered}
$$

In Eqns. 7-12, $\sigma$ is the total number of elements $(\mathrm{C}, \mathrm{H}, \mathrm{O}$ and $\mathrm{N}$ ) in the reactor; $N_{s}$ is the total number of species used by the chemical mechanism; $N_{v}$ is the total number of virtual species; $N$ is the total number of elements $(\mathrm{C}, \mathrm{H}, \mathrm{O}$ and $\mathrm{N})$ in each chemical species; $x$ refers to mole fractions; $Y$ refers to mass fractions; $h$ is the mass specific enthalpy; $c_{p}$ is the mass specific heat. The virtual species approach was presented and validated by the authors in a previous work [24]. Constant-volume and constant pressure reactor calculations performed in a wide range of conditions made possible to verify that cumulative heat release, reactor temperature and pressure evolutions are consistent with the cases where the full set of species is used. No unphysical results with negative species were found.

The table also includes the mass fractions of chemical species which are of interest for the user ( $Y_{o}$ in Fig. 1), either for post-processing reason or because they are relevant for the formation of the main pollutants and have to be used by the related sub-models.

\section{Tabulated well mixed model (TWM)}

The operation of the tabulated well-mixed model is reported in Fig. 2. In the CFD domain, transport equations for mixture fraction, enthalpy, unburned gas temperature and progress variable are solved and then the table is accessed with the local cell values. Interpolation of table values at cell conditions is performed by means of an inverse, distance weighted technique. Mixture fraction equation includes fuel evaporation:

$$
\frac{\partial \rho Z}{\partial t}+\nabla(\rho \mathbf{U} Z)-\nabla\left(\mu_{t} \nabla Z\right)=\dot{S}_{Z}
$$

The progress variable source term used in the $C$ transport equation is derived from Eq. 6 :

$$
\begin{gathered}
\frac{\partial \rho C}{\partial t}+\nabla(\rho \mathbf{U} C)-\nabla\left(\frac{\mu_{t}}{S c_{t}} \nabla C\right)=\rho \dot{C} \\
\dot{C}=\left(C_{\text {max }}-C_{\text {min }}\right) \cdot \dot{c}
\end{gathered}
$$

To consistently access the table data, it is necessary to solve an additional equation for the unburned gas enthalpy which is then used to estimate the unburned gas temperature $T_{u}$ which is one of the independent variables of the table:

$$
\frac{\partial \rho h_{u}}{\partial t}+\nabla\left(\rho \mathbf{U} h_{u}\right)-\nabla\left(\alpha_{t} \nabla h_{u}\right)=\dot{Q}_{s}+\frac{\rho}{\rho_{u}} \cdot \frac{D p}{D t}
$$

where $\alpha_{t}$ is the turbulent thermal diffusivity and $\rho_{u}$ is the density of unburned gases which is computed from cell pressure, chemical composition at $C=0$ and $T_{u} \cdot \dot{Q}_{s}$ is the term related to spray evaporation which assumes different values in case the mixing line is assumed or not in the table generation process.

It is expected that both in case of direct-integration and tabulated kinetics the well mixed model produces similar results. However, it must be pointed out that tabulation introduces significant simplifications since a $N_{s}+2$ dimensional problem is then reduced only to four dimensions. This aspect mainly affects the ignition process when $C$ is diffused to rich mixtures $(\phi>3)$ which are characterized by a long ignition delay and a single stage very fast ignition. As a consequence, rich regions will be ignited almost instantaneously and high values of $C$ will be diffused back to lean or stoichiometric mixtures leading to a very anticipated auto-ignition. To avoid this, reaction rates are set to zero in regions where dual-stage ignition does not happen, corresponding approximately to $\phi=3$. This aspect might have some effects on prediction of soot emissions since inception and surface grow phases take place where the mixture is very rich. In terms of ignition delay, increasing the maximum equivalence ratio value at which reaction rates are computed from 3 to 4 will reduce the ignition delay by a factor of $25 \%$, leading to a significant underestimation of experimental data.

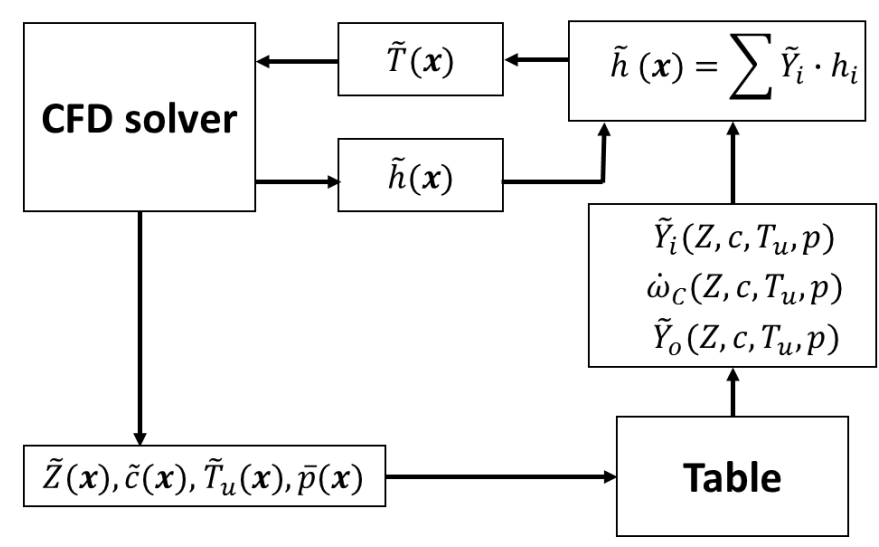

Figure 2: Operation of the tabulated well-mixed combustion model (TWM).

\section{Tabulated presumed PDF combustion model (TPPDF)}

This approach was applied in $[17,27]$ to simulation of compression-ignition engines: turbulence chemistry interaction is incorporated in the computation of the progress variable source term, assuming a probability density function for both progress variable and mixture fraction as follows:

$$
\dot{C}=\int_{0}^{1} \int_{0}^{1} \dot{C}\left(p, T_{u}, Z, c\right) p\left(Z, \widetilde{Z^{\prime \prime 2}}\right) p\left(c, \widetilde{c^{\prime \prime 2}}\right) d c d Z
$$


since this work is mainly focused on diffusion combustion, a $\delta$-PDF distribution was assumed for the progress variable while a $\beta$-PDF function was used for the mixture fraction. This requires to additionally solve the mixture fraction variance $\widetilde{Z^{\prime \prime 2}}$ equation:

$\frac{\partial \rho \widetilde{Z^{\prime \prime 2}}}{\partial t}+\nabla\left(\rho \mathbf{U} \widetilde{Z^{\prime \prime 2}}\right)-\nabla\left(\mu_{t} \nabla \widetilde{Z^{\prime \prime 2}}\right)=2 \frac{\mu_{t}}{S c}|\nabla Z|^{2}-\rho \chi$

The sink term appearing in Eq. 19 is the average scalar dissipation rate, which is function of the turbulent time scale and mixture fraction variance:

$$
\chi=C_{\chi} \frac{\varepsilon}{k} \widetilde{Z^{\prime \prime 2}}
$$

To conveniently apply this model to engine simulations without introducing significant computational overheads, the table based on homogeneous reactor is processed to include the effects of turbulence chemistry interation as shown in Fig. 3. In particular, the user specifies ranges of mixture fraction and progress variable segregation factors ( $S_{Z}$ and $S_{c}$, respectively), defined as the ratio between variance and maximum variance values:

$$
\begin{aligned}
S_{Z} & =\frac{\widetilde{Z^{\prime \prime 2}}}{Z(1-Z)} \\
S_{c} & =\frac{\widetilde{c^{\prime \prime 2}}}{c(1-c)}
\end{aligned}
$$

for any value of $Z, S_{Z}, c$ and $S_{c}$ it is possible to compute variances and the coefficients for the probability density function distributions. On the basis of them, integration is performed for all the tabulated quantities $f_{H R}$ and a new table including the effects of mixture fraction and progress variable fluctuations is available for the presumed PDF combustion model.

$$
f_{\text {seg }}=\int_{0}^{1} \int_{0}^{1} f_{H R}\left(p, T_{u}, Z, c\right) p\left(Z, \widetilde{Z^{\prime \prime 2}}\right) p\left(c, \widetilde{c^{\prime \prime 2}}\right) d c d Z
$$

Compared to other widely used combustion models for non-premixed combustion, the TPPDF model does not consider any sub-grid diffusion in the mixture fraction space, while other approaches does either through a suitable mixing model [28] or by means of the scalar dissipation rate term. Turbulence/chemistry interaction with the presumed PDF combustion model is expected to reduce the maximum flame temperatures and to increase the thickness of the flame compared to the well-mixed model.

\section{Tabulated representative interactive flamelet model (TRIF)}

This model is based on the laminar flamelet concept, assuming that the smallest turbulent time and length scales are much larger than the chemical ones and there exists a

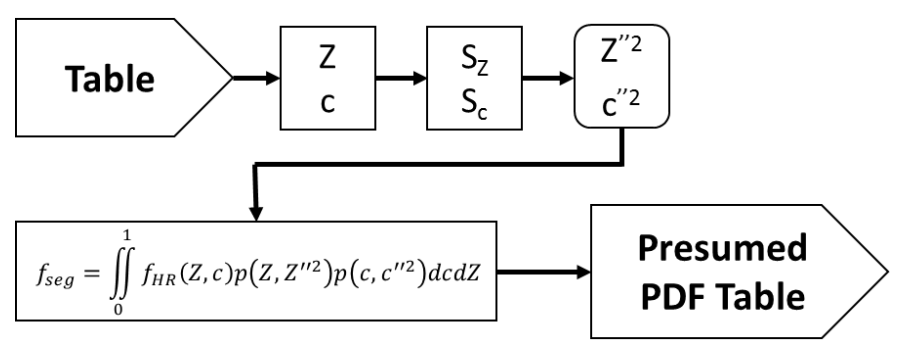

Figure 3: Generation of the chemistry table used for the presumed PDF combustion model (TPPDF).

locally undisturbed sheet where reactions occur [7]. This sheet can be treated as an ensemble of stretched counterflow diffusion flames, called flamelets. The advantage of such treatment is that all reacting scalars only depend on the mixture fraction variable, $Z$, which is related to the local fuel-to-air ratio for non-premixed combustion. Hence, local chemical composition can be estimated from the $Z$ field in the CFD domain, assuming that its sub-grid distribution can be represented by a $\beta$-pdf. In order to properly account for local flow and turbulence effects on the flame structure and predict flame stabilization, a multiple number of flamelets can be used. Each one is representative of a certain portion of the injected fuel mass, and chemical composition in each cell is computed from mixture fraction and flamelet marker distribution as follows:

$$
Y_{i}(\vec{x})=\sum_{j=1}^{N_{f}} M_{j} \int_{0}^{1} Y_{j, i}(\tilde{Z}) P\left(\tilde{Z}, \widetilde{Z^{\prime \prime 2}}\right) d Z
$$

For each flamelet marker, the following transport equation is solved:

$$
\frac{\partial \rho \tilde{M}_{j}}{\partial t}+\nabla\left(\rho \mathbf{U} \tilde{M}_{j}\right)-\nabla \cdot\left(\frac{\mu_{t}}{S c_{Z}} \nabla \tilde{M}_{j}\right)=\dot{S}_{M_{j}}
$$

where the source term $\dot{S}_{M_{j}}$ corresponds to $\dot{S}_{Z}$ only for a specified interval of the injection duration, while it is zero elsewhere. Flamelet markers must also satisfy the following relation:

$$
Z=\sum_{j=1}^{N_{f}} M_{j}
$$

The local flame structure is defined by the flamelet equations that are solved for the progress variable $C$ and enthalpy assuming unity Lewis number [7] in the mixture fraction space:

$$
\begin{aligned}
\rho \frac{\partial C}{\partial t} & =\rho \frac{\chi_{z}}{2} \frac{\partial^{2} C}{\partial Z^{2}}+\dot{C} \\
\rho \frac{\partial h}{\partial t} & =\rho \frac{\chi_{z}}{2} \frac{\partial^{2} h}{\partial Z^{2}}+\frac{d p}{d t}
\end{aligned}
$$

The chemical composition in the mixture fraction space is retrieved from the chemistry table in the same way as done 
for the TWM model in the CFD domain. Effects of mixing related to turbulence and flow-field are grouped into the scalar dissipation rate term $\chi_{z}$ which is function of the scalar dissipation rate at stoichiometric mixture fraction conditions $\widehat{\chi_{s t}, j}$ which is computed for each flamelet.

The Fig. 4 summarizes the operation of the TRIF combustion model, illustrating the mutual interactions between the CFD, flamelets domains and chemistry table. At each timestep, average stoichiometric scalar dissipation rate values are passed to each flamelet, that solves Eqn. 27-28 accordingly. Source term for progress variable equation is computed from the chemistry table and is function of $C$ and local thermodynamic conditions in the mixture fraction space. To avoid too early ignition due to progress variable diffusion from the rich to the lean mixture, also here progress variable reaction rate is set to zero for mixture fraction values corresponding to equivalence ratio higher than 3. The chemical composition in the CFD domain is computed from the mixture fraction, its variance and the flamelet marker distribution. Temperature is updated from new chemical composition and total enthalpy, whose variation is only due to flow and spray evaporation. The approach used in this work was applied by [22] to model diffusion flames. Compared to the tabulation of unsteady flamelets, TRIF employs a table of smaller sizes since neither scalar dissipation rate and mixture fraction segregation have to be tabulated. However, it will have higher computational costs due to the need to perform on-line the integration of Eq. 24 in any computational cell for any flamelet [5].

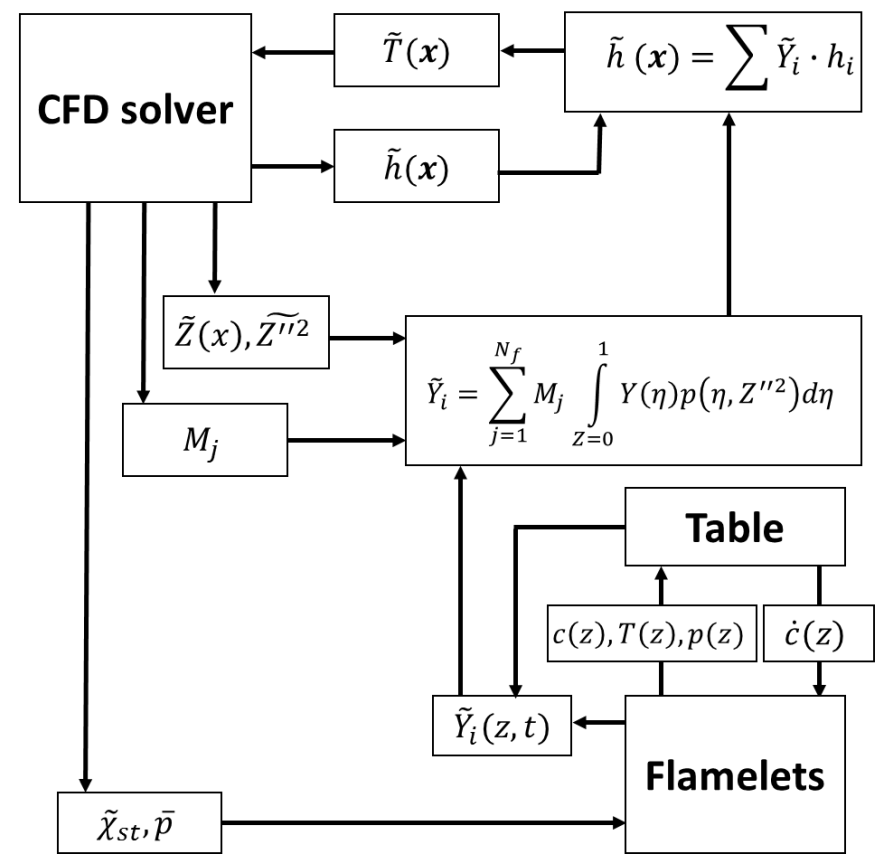

Figure 4: Operation of the tabulated representative interactive flamelet combustion model (TRIF).

\section{Pollutant emissions}

Suitable sub-models to estimate the main pollutant emissions formed during the combustion process were also incorporated. In particular, the possibility to predict $\mathrm{NO}_{x}$ and soot was included.

$\mathrm{NO}_{x}$ emissions are considered to be only $\mathrm{NO}$ and such species is assumed to be formed with the Extended Zeldovich mechanism as follows:

$$
\begin{aligned}
N+N O & \leftrightarrow N_{2}+O \\
N+O_{2} & \leftrightarrow N O+O \\
N+O H & \leftrightarrow N O+H
\end{aligned}
$$

Reaction rate constants are taken from [29] after verifying that suggested values from recent works produce very similar results. A transport equation for $N O$ mass fraction $Y_{N O}$ is solved in the CFD domain with a source term computed in any cell accounting for the average temperature and species concentrations except $N O$ for which the local concentration taken from $Y_{N O}$ is used.

Soot emissions were estimated by means of the semiempirical model proposed by Lindstedt and co-workers [30]: two transport equations for soot particle number density $N_{p}$ and volume fraction $f_{v}$ are solved, with source terms related to nucleation, coagulation, surface growth and oxidation processes as follows:

$$
\begin{aligned}
\dot{\omega}_{N_{p}} & =\dot{\omega}_{\text {inc }}-\dot{\omega}_{\text {coag }} \\
\dot{\omega}_{f_{v}} & =\dot{\omega}_{\text {inc }}+\dot{\omega}_{\text {grow }}-\dot{\omega}_{o x i, O_{2}}-\dot{\omega}_{o x i, O H}
\end{aligned}
$$

Inception and surface growth source terms $\left(\dot{\omega}_{i n c}\right.$ and $\dot{\omega}_{\text {grow }}$, respectively) depend linearly on the soot precursor concentration which is considered to be acetylene, consistently with other papers in which the same model was successfully applied to Diesel combustion [31]. As it has been experimentally observed, the reactivity of the soot particles decreases in time; in this work, to be consistent with the original study from Lindstedt [30], this aspect was accounted for by assuming the soot surface growth rate proportional to the square root of the specific surface area, $S_{\text {soot }}$. Coagulation of soot particles, $\dot{\omega}_{\text {coag }}$, is modeled using the normal square dependence [30]. Soot oxidation depends on $\mathrm{O}_{2}$ and $\mathrm{OH}$ concentrations, following [30]. It is well known that the formation of soot is rather a slow process compared to the other species involved in the combustion chemistry, thus the fast chemistry assumption, solving $f_{v}$ and $N_{p}$ equations in the mixture fraction space might be questionable when it is necessary to predict the effects of mixture distribution, injection pressure and combustion chamber geometry [32]. For this reason transport equations for the soot model are solved in the CFD domain and source terms are computed on the basis of the local species concentrations and thermodynamic conditions. 


\section{Experimental validation}

Experiments carried out in a constant-volume vessel (Spray A) and a Medium Duty (MD) engine were used to assess and validate the proposed combustion models based on tabulated kinetics. Simulations were carried out using the standard k-epsilon turbulence model with the round jet correction $\left(C_{1}=1.5\right)$ and the Lagrangian approach was used to describe the spray evolution. The KHRT model was employed to predict primary and secondary breakup [33] and the proposed setups for spray model was validated by the authors in [5] under non-reacting, evaporating conditions.

Diesel fuel was assumed to be n-dodecane and in this work its oxidation was modeled using the mechanism proposed by Frassoldati et al. [34]. It has 96 species and 993 reactions and it was extensively validated with experimental data in a wide range of conditions including flow and stirred reactor experiments, autoignition delay times, laminar flame speeds, and autoignition of isolated fuel droplets in microgravity conditions. Due to its acceptable size, this mechanism can be also employed for simulations using direct chemistry integration whose results will be also shown in this work to verify the consitency of the proposed approaches.

\section{Spray-A}

The Spray-A experiment from Engine Combustion Network was used for model assessment and preliminary validation [35]. $\mathrm{N}$-dodecane fuel is delivered through a single hole nozzle $(\mathrm{d}=90 \mu \mathrm{m})$ in a constant-volume vessel where ambient conditions are similar to those found at start of injection (SOI) time in Diesel engines. Different measurement techniques make possible to fully characterize the combustion process in terms of heat release rate, lift-off length and distribution of soot $[36,37,38]$.

Simulated operating conditions are reported in Tab. 1, they include variation of ambient temperature and oxygen concentration with a $22.8 \mathrm{~kg} / \mathrm{m}^{3}$ ambient density. Fuel is injected at a 1500 bar pressure for a $5 \mathrm{~ms}$ duration. A 2D, axy-symmetric grid was used with the same vessel height (108 $\mathrm{mm}$ ) while the mesh radius was adjusted to properly match the measured vessel volume.

\begin{tabular}{|c|c|c|c|}
\hline & $\mathrm{O} 2[\%]$ & $\mathrm{T}[\mathrm{K}]$ & $\rho\left[\mathrm{kg} / \mathrm{m}^{3}\right]$ \\
\hline Baseline & 15 & 900 & 22.8 \\
\hline Low-T & 15 & 800 & 22.8 \\
\hline High-T & 15 & 1100 & 22.8 \\
\hline O2-13 & 13 & 900 & 22.8 \\
\hline O2-21 & 21 & 900 & 22.8 \\
\hline
\end{tabular}

Table 1: Simulated operating points for the ECN spray-A experiment.

Three chemistry tables were generated, one for each tested amount of oxygen concentration. The oxidizer composition is the same as the one reported experimentally. All the tables consider a $750-1100 \mathrm{~K}$ temperature range, 25 points in the mixture fraction space and two pressure levels corresponding to the minimum and maximum ones encountered in the considered conditions. Details about table discretization are reported in Tab. 2. Initial temperature conditions for homogeneous reactor calculations include fuel evaporation effects, according to Eq. 1.

\begin{tabular}{cc}
\hline Temperature $[\mathrm{K}]$ & $750,800,850,900$ \\
& $950,1000,1050,1100$ \\
\hline Equivalence ratio & $0.4,0.5,0.6,0.7,0.85$ \\
& $0.9,0.95,1.00,1.05,1.1$ \\
& $1.15,1.2,1.25,1.35,1.45$ \\
& $1.55,1.65,1.75,1.9,2$ \\
& $2.10,2.30,2.5,2.7,3$ \\
\hline Pressure [bar] & $50-80$ \\
\hline
\end{tabular}

Table 2: Chemistry table discretization used for the simulation of the spray-A experiment.

First, the baseline condition was analyzed and the performance of the TWM and TRIF models were compared with the corresponding approaches where direct-integration is used: DIWM and DIRIF, respectively. Temperature evolution inside the domain in terms of $Z-T$ diagrams for DIWM and TWM models are shown in Figs. 5-6. For the sake of clarification, both figures report also the stoichiometric mixture fraction $Z_{s t}$ and Fig. 6 also the maximum mixture fraction value $Z_{\max }$ which is used to compute the progress variable source term.

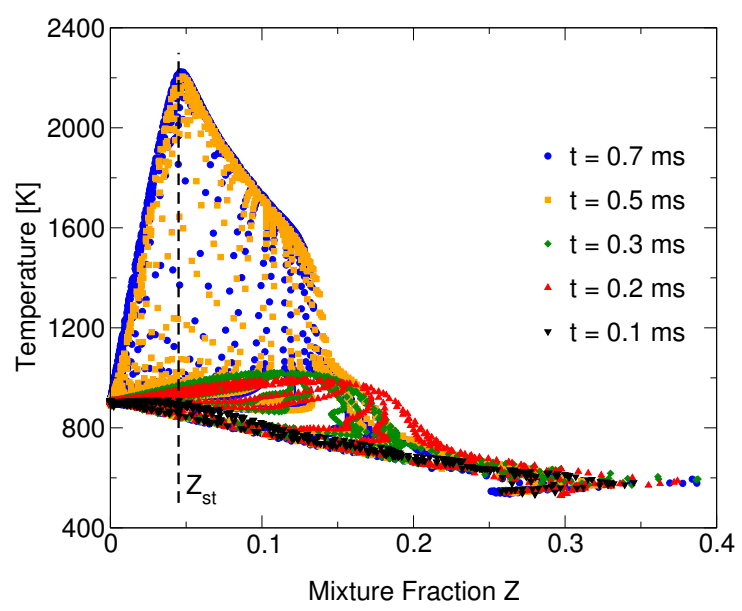

Figure 5: Ignition process for the Spray-A baseline condition reported for Direct Integration Well Mixed Model

For both models, during the ignition phase, heat starts to be released in the lean side, peak temperatures are located in the rich mixture region $\left(Z>Z_{s t}\right)$ during the 0.2-0.3 $\mathrm{ms}$ time interval and ignition is almost completed after 0.5 ms. Tabulated kinetics has a higher reactivity compared to direct-integration and this is due to the progress variable diffusion where the mixture is rich, while the opposite seems to happen when using direct integration where the 
main effect seems to be the slowdown of chemical reactions due to the diffusion of the unburned fuel.

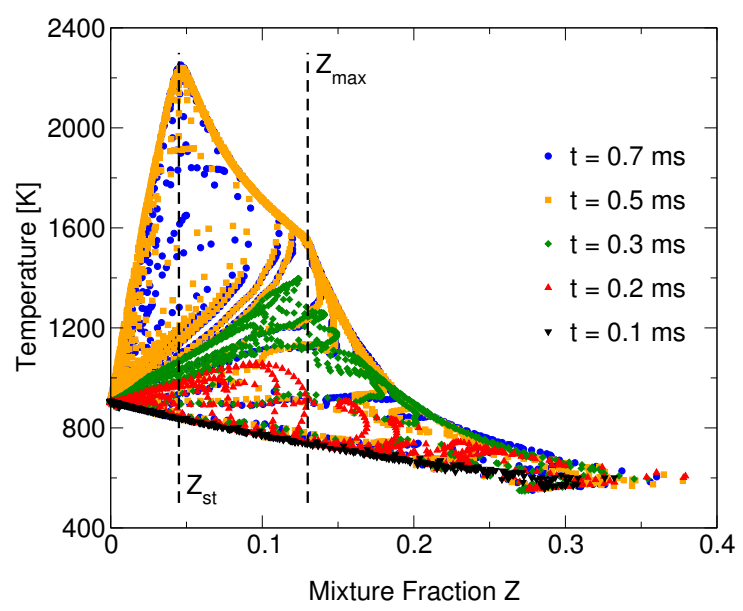

Figure 6: Ignition process for the Spray-A baseline condition reported for Tabulated Chemistry Well Mixed Model (TWM).

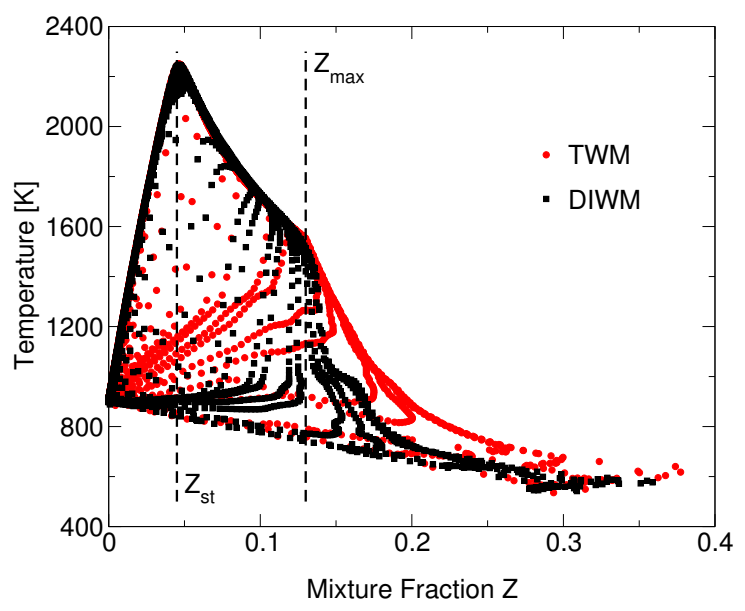

Figure 7: Spray-A baseline condition: temperature distribution after $1.5 \mathrm{~ms}$ for DIWM and TWM models.

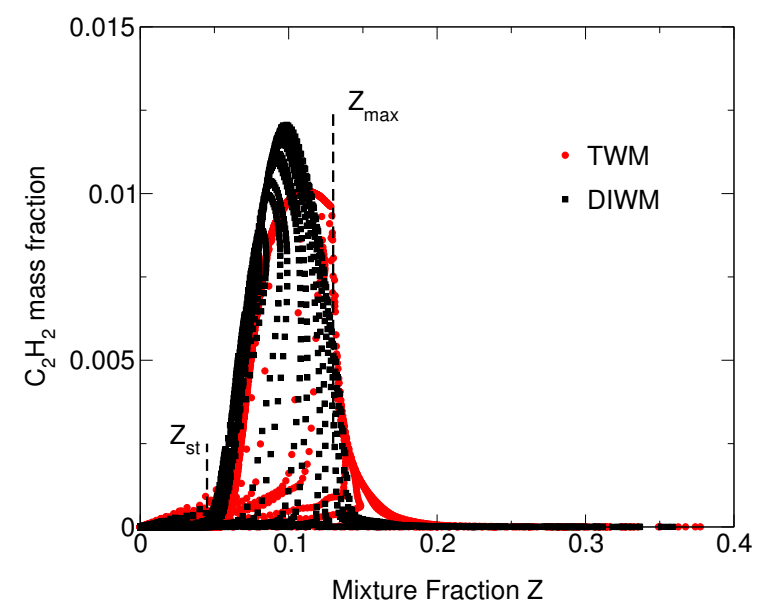

Figure 8: Spray-A baseline condition: acetylene $\left(\mathrm{C}_{2} \mathrm{H}_{2}\right)$ distribution after $1.5 \mathrm{~ms}$ for DIWM and TWM models.

When diffusion flame structure is fully established at 1.5 ms, Fig. 7 illustrates that both models produce very similar $Z-T$ scatters with maximum temperature located where the mixture is stoichiometric. Diffusion of progress variable affects the temperature distribution in the rich region, and also the homogeneous reactor assumption produces a slight difference in the acetylene distribution (see Fig. 8) which is used as soot precursor in this work. In particular, maximum $C_{2} H_{2}$ mass fraction is at $Z=0.1$ for the DIWM model while for TWM it is found at $Z=0.11$ with a lower value and this difference might be responsible for different amout of soot mass produced when using these two different combustion models.

Fig. 9 illustrates computed vessel pressure rises and heat release rates (HRR) for DIWM and TWM models. Consistently with Figs. 5-6, both approaches produce similar HRR profiles during ignition with a larger cool flame peak from TWM model. During the mixing controlled combustion phase, predicted HRR values are very similar. The corresponding vessel pressure are also in very good agreement, showing that the proposed virtual species approach properly reproduces the main thermodynamic properties of the full set of species employed in the detailed kinetic mechanism.

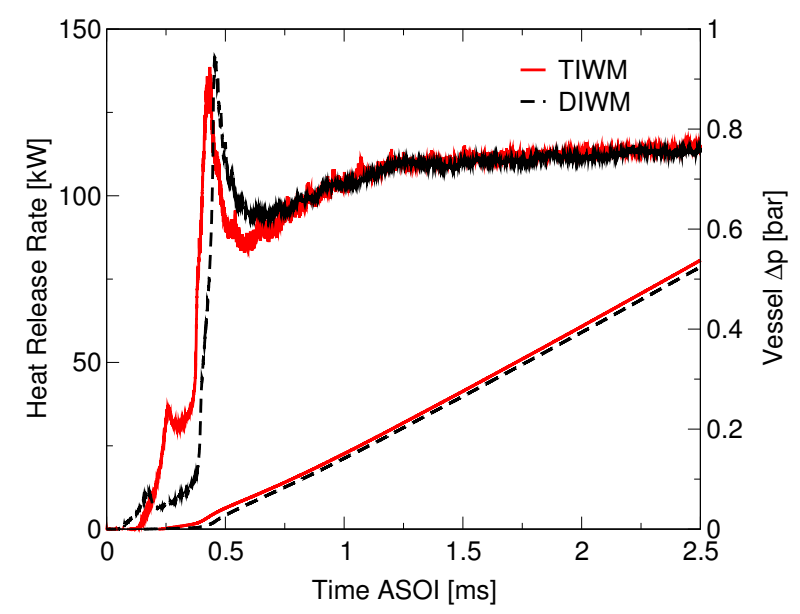

Figure 9: Vessel heat release rate and pressure increment as function of DIWM and TWM combustion models.

Finally, Fig. 10 reports a comparison between computed temperature distributions from TWM and DIWM models. The same figure reports also iso-contours of stoichiometric mixture fraction with the greeen line and a dashed white line located where experimental lift-off length was found. As already discussed in past works [5, 15], the well-mixed model with direct chemistry integration overestimates the lift-off since it does not consider turbulence-chemistry interaction and, because of this assumption, there is a larger region where the scalar dissipation rate is higher than the value at which diffusion flame extinguishes. In the TWM model, the flame has a shorter lift-off length because of the higher mixture reactivity where the mixture is rich, as already discussed in Figs. 5-6. Diffusion of progress variable from rich to stoichiometric mixture reduces the length at 
which the flame stabilized. This aspect requires to be improved, and will be matter of investigation in a future work.

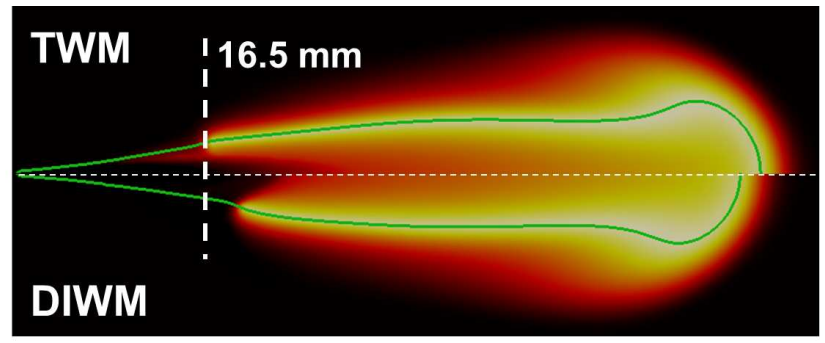

Figure 10: Computed temperature distribution for the DIWM and TWM models. Temperature range: 900 (black) - 2300 (yellow).

In terms of computational costs, the TWM model approximately requires 20 times less than what is needed by direct chemistry integration in combination with multi-zone chemistry [13]. Considering also the very similar results produced by both models in terms of flame structure, ignition delay, vessel pressure and heat release rate, it is possible to conclude that the TWM model can represent an accurate and computationally efficient solution for combustion simulations.

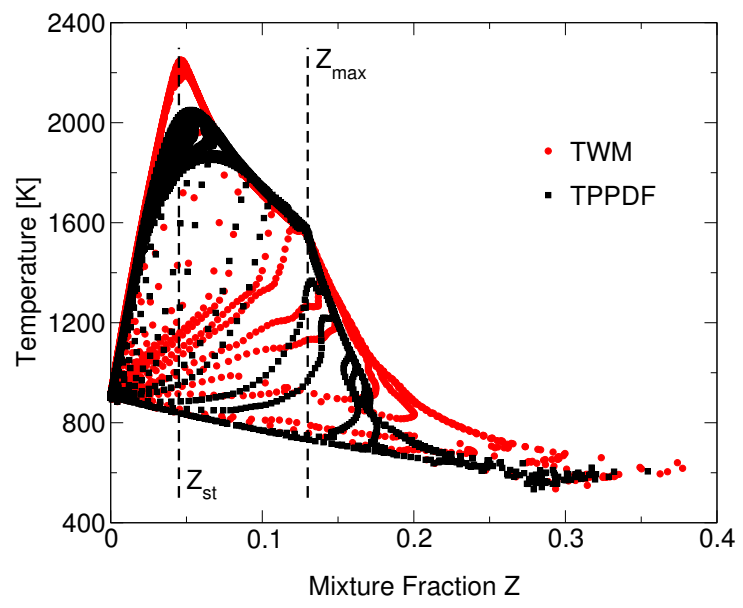

Figure 11: Spray-A baseline condition: temperature distribution after $1.5 \mathrm{~ms}$ for DIWM and TWM models.

To overcome the limitation of the TWM model which is represented by the neglection of turbulence-chemistry interaction, the TPPDF model computes the average progress variable reaction rate by integrating the corresponding homogeneous reactor values with a probability density function in each CFD cell. It is not possible to implement the same approach with direct integration, hence results of the TPPDF model will be compared with the ones from TWM model. Fig. 11 reports the computed temperature distribution as function of the mixture fraction for the TWM and TPPDF models. As expected, most of the changes are located close to the stoichiometric region where averaged cell composition also includes both lean and rich combustion products with a consequent reduction of the maximum temperatures. Concerning heat release rate and vessel pres- sure profiles, Fig. 12 shows that inclusion of turbulencechemistry interaction reduces both the heat release rate and ignition delay. While the first is related to the new vessel temperature distribution, the second is the consequence of an enhanced reactivity due to the way the averaged progress variable source term is computed, including contributions from the whole mixture fraction space also for very lean and rich mixtures. Computed flame structures from TWM and TPPDF models are finally reported in Fig. 13. Shorter ignition delay and higher reactivity are responsable for shorter lift-off length values when turbulence-chemistry interaction is included in tabulated kinetics using the presumed PDF approach.

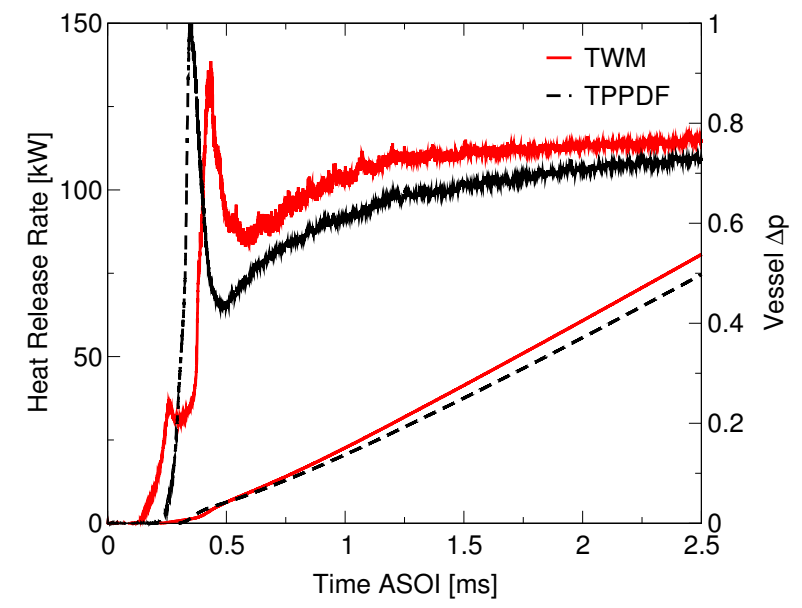

Figure 12: Vessel heat release rate and pressure increment as function of TWM and TPPDF combustion models.

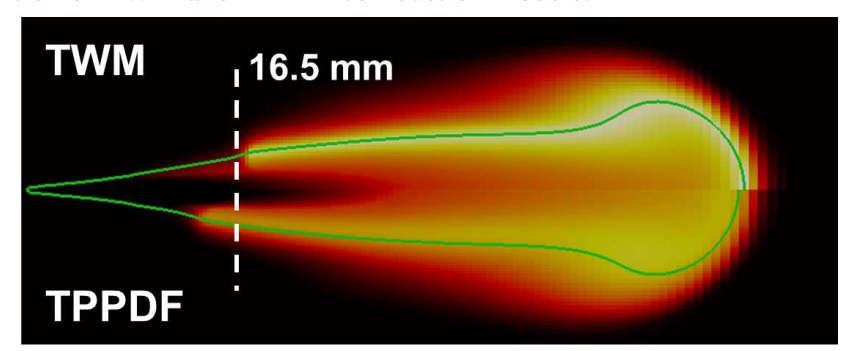

Figure 13: Computed temperature distribution for the TWM and TPPDF models. Temperature range: 900 (black) - 2300 (yellow).

Effects of ambient temperature and oxygen concentration on ignition delay (ID) and flame lift-off length (LOL) are presented in Figs. 14-15 for the models based on tabulated kinetics and for the DIWM one which is used as reference. TWM and DIWM models have an acceptable agreement with experimental data of ignition delay either as function of ambient oxygen concentration and ambient temperature. Concerning LOL, shorter values computed with tabulated kinetics seem to be related to the diffusion of progress variable leading to a faster stabilization compared to what happens with direct-integration. However, experimental trends are correctly captured. Results from the TWM model are the closest to measured data, but at this point it is not possible to conclude that it is the best approach. Turbulence- 
chemistry interaction effects seem to be important for the $13 \%$ and $15 \%$ oxygen concentration cases, where the LOL value computed by TPPDF is lower than experimental data and the one estimated by the TWM model. This is probably due to the way the $\beta-P D F$ is integrated assuming constant progress variable in the mixture fraction space which does not completely consider the ignition history of the different reactors. The possibility to introduce the progress variable variance to see if results can be improved will be a matter of a future work.
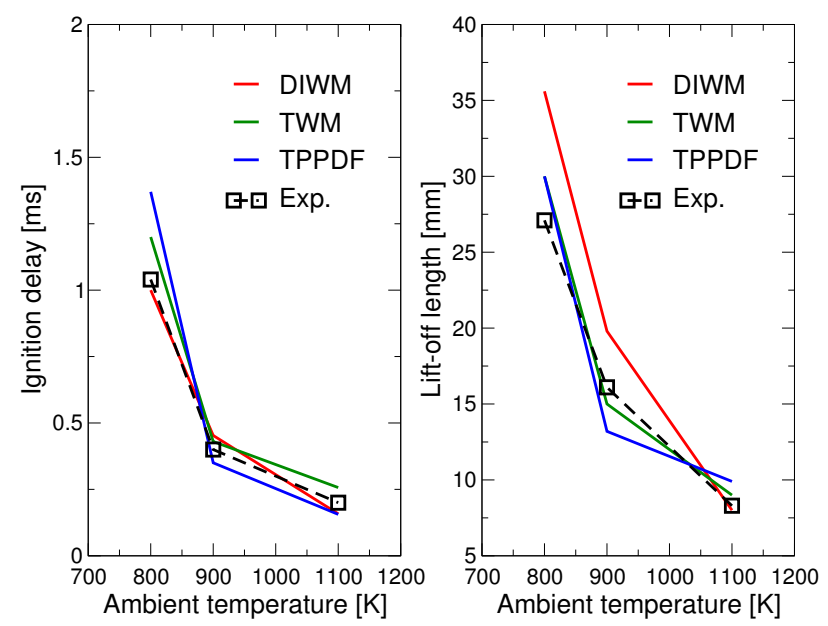

Figure 14: Comparison between experimental data and computed ones from TWM, DIWM and TPPDF models as function of ambient temperature: (a) Ignition delay; (b) Flame-lift off.
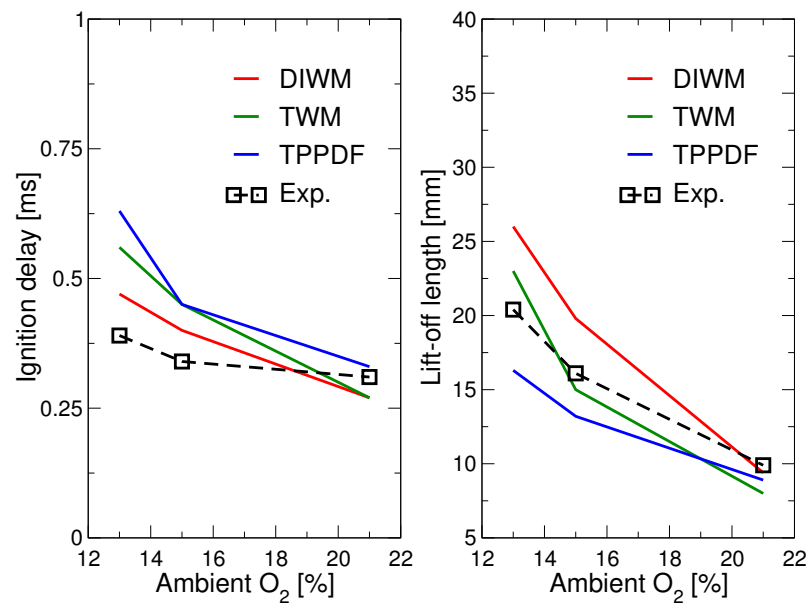

Figure 15: Comparison between experimental data and computed ones from TWM, DIWM and TPPDF models as function of ambient oxygen concentration: (a) Ignition delay; (b) Flame-lift off.

The use of the homogeneous reactor assumption to estimate reaction rates in the representative interactive flamelet model is illustrated in Figs. 16(a)-(b) where temperature profiles in the mixture fraction space are illustrated for the baseline condition at different instant after start of injector for the DIRIF and TRIF model. In both cases, simulations were carried out with a single flamelet. Even with a changed flame structure assumption, diffusion of progress variable from rich to stoichiometric mixture fraction is still responsible for the differences between computed TRIF and DIRIF temperature distributions in the mixture fraction space. In both the cases the ignition process is completed after $0.5 \mathrm{~ms}$, and limitation of reaction rate to values lower than $\phi=3$ makes the high temperature region narrower for the TRIF model compared to DIRIF. In terms of computational efficiency, TRIF is five times faster than DIRIF. The reduced speed-up factor with respect to the well-mixed model cases is due to the fact that, in the DIRIF, chemistry is integrated in a limited number of points in the mixture fraction space and also because both approaches perform on-line integration of the $\beta$-PDF to compute the average cell composition. (a) RIF

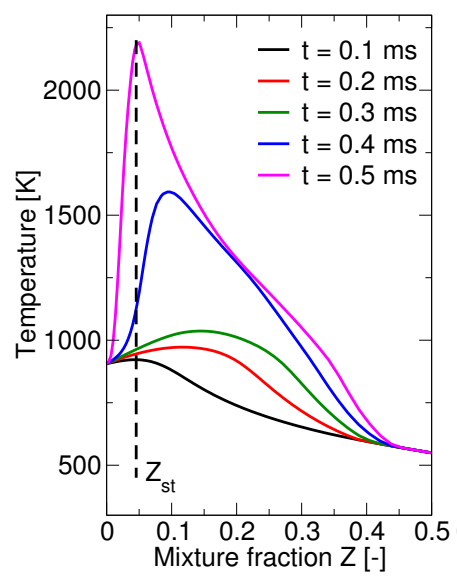

(b) TRIF

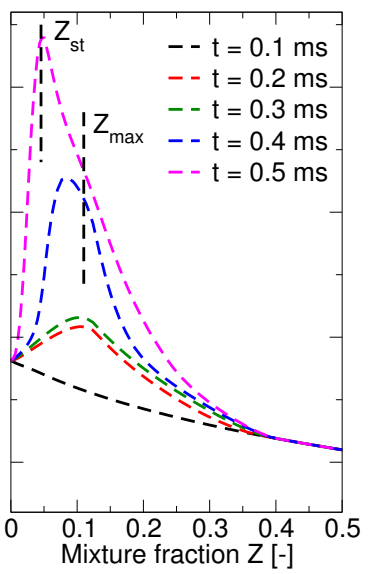

Figure 16: Temperature mixture fraction profiles during the ignition process: (a) DIRIF model; (b) TRIF model.

Fig. 17 illustrates the behavior of the TRIF model in terms of heat release rate and vessel pressure during auto-ignition and mixing-controlled combustion model compared to the case where direct-integration is used. Ignition takes place at a similar instants and then the TRIF behavior during the mixing controlled combustion is aligned with DIRIF.

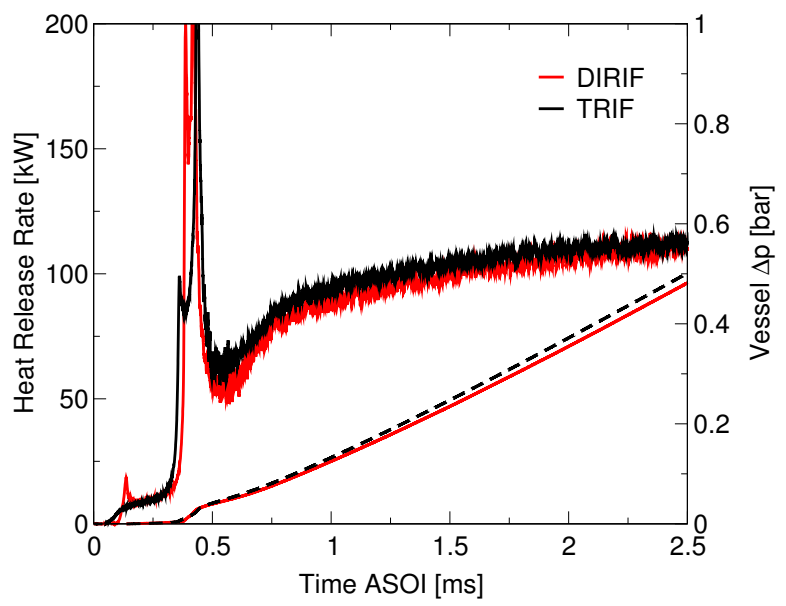

Figure 17: Vessel heat release rate and pressure increment from DIRIF and TRIF combustion models.

Finally, Fig. 18 reports a comparison between computed 
and experimental values of ignition delay for the TRIF and DIRIF models in the different tested operating conditions. Since only one flamelet was used, flame is stabilized directly at the nozzle exit after ignition. Tabulated kinetics is in good agreement with experimental data, except for the $13 \% \mathrm{O}_{2}$ case, probably because of the neglected contribution to ignition process from rich mixtures where there is a high level of dilution. This aspect will be matter of investigations in a future work.
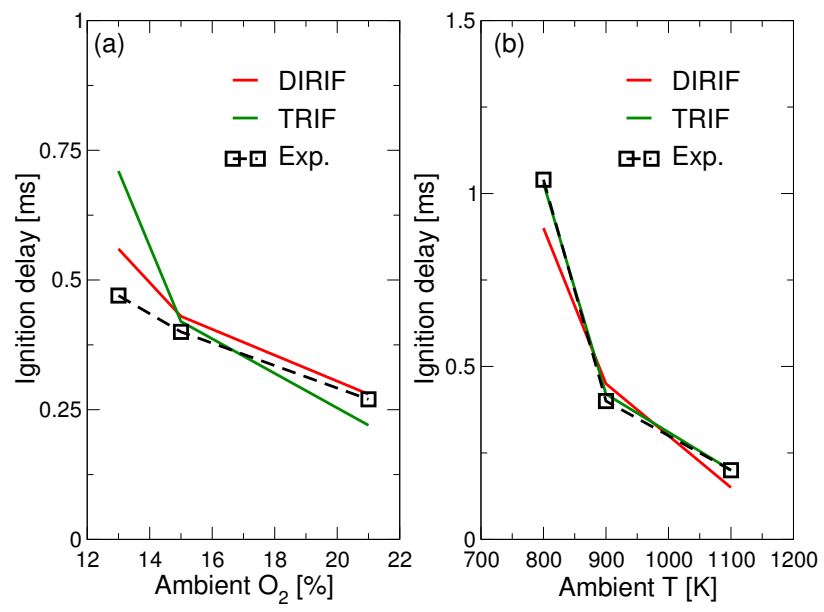

Figure 18: Ignition delay comparison between experimental data and computed ones from TRIF and DIRIF models as function: (a) Ambient oxygen concentration; (b) Ambient temperature.

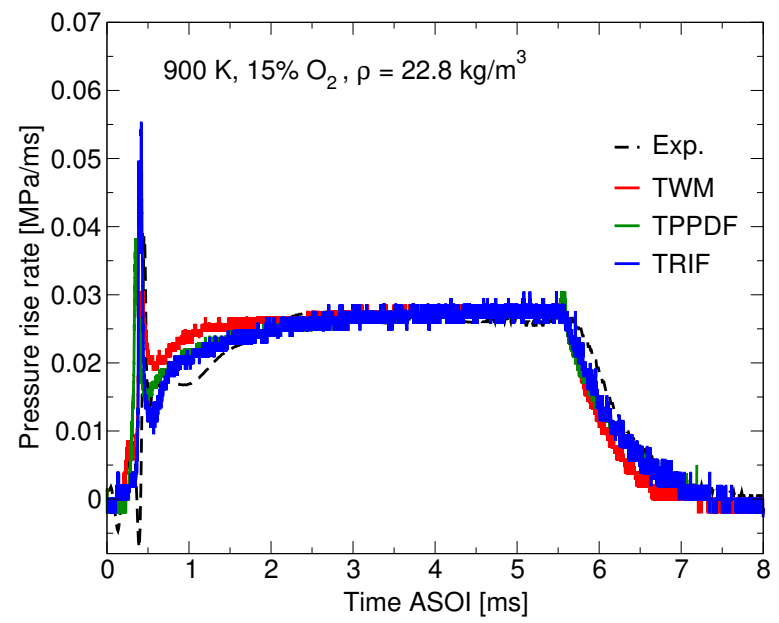

Figure 19: Comparison between experimental and computed pressure rise rate $(d p / d t)$ using the TWM, TPPDF and TRIF models at $900 \mathrm{~K}, 15 \% \mathrm{O}_{2}$

In engine simulations, prediction of pressure trace is of great importance in order to properly predict performance and efficiency. For this reason, Figs. 19-23 report a comparison between experimental and computed values of pressure rise rate $d p / d t$ as function of ambient temperature and oxygen concentration, respectively. In all the simulated conditions, it is possible to see that when combustion is mainly mixing-controlled (in the $2-5 \mathrm{~ms}$ time interval), all the three models based on tabulated kinetics predict the same $d p / d t$. Concerning turbulence-chemistry interaction, its main role seems to be in the transition from auto-ignition (identified with the $d p / d t$ peak) to mixing-controlled combustion. Finally, the behavior of the TPPDF model is similar to TRIF, despite there is no assumed flame structure behind. This aspect can be clearly seen for all the operating conditions, except the $800 \mathrm{~K}$ one, where the TPPDF ignites much later than the other two approaches.

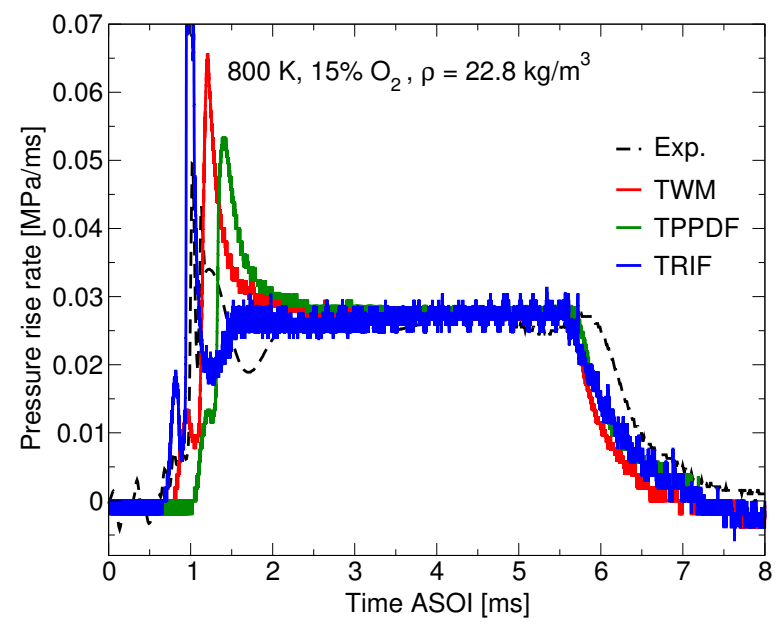

Figure 20: Comparison between experimental and computed pressure rise rate $(d p / d t)$ using the TWM, TPPDF and TRIF models at $800 \mathrm{~K}, 15 \% \mathrm{O}_{2}$

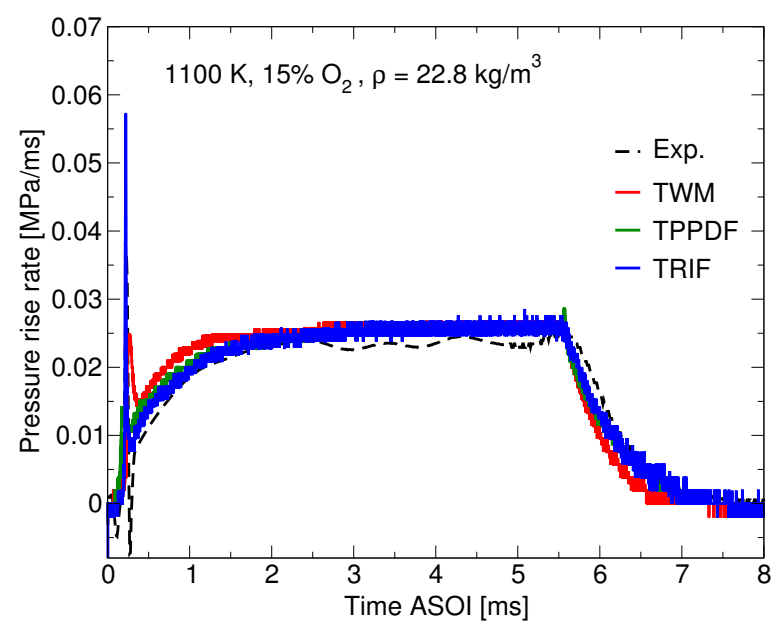

Figure 21: Comparison between experimental and computed pressure rise rate $(d p / d t)$ using the TWM, TPPDF and TRIF models at $1100 \mathrm{~K}, 15 \% \mathrm{O}_{2}$

Recently, diffused, back-illumination, extinction imaging (DBIEI) was used to acquire time-resolved images of soot optical thickness $(K L)$ in n-dodecane spray combustion experiments [36]. DBIEI maps of KL are processed to obtain the dimensional extinction coefficient $K$, which can then be related to the radial distribution of the soot volume fraction $f_{v}$. Assuming an axi-symmetric jet, the evolution of the total amount of soot can be estimated directly from $f_{v}$. In particular, experimental data were available for four over the five tested operating conditions making possible to understand the capability of the proposed models to predict soot emissions at different ambient oxygen concentrations 


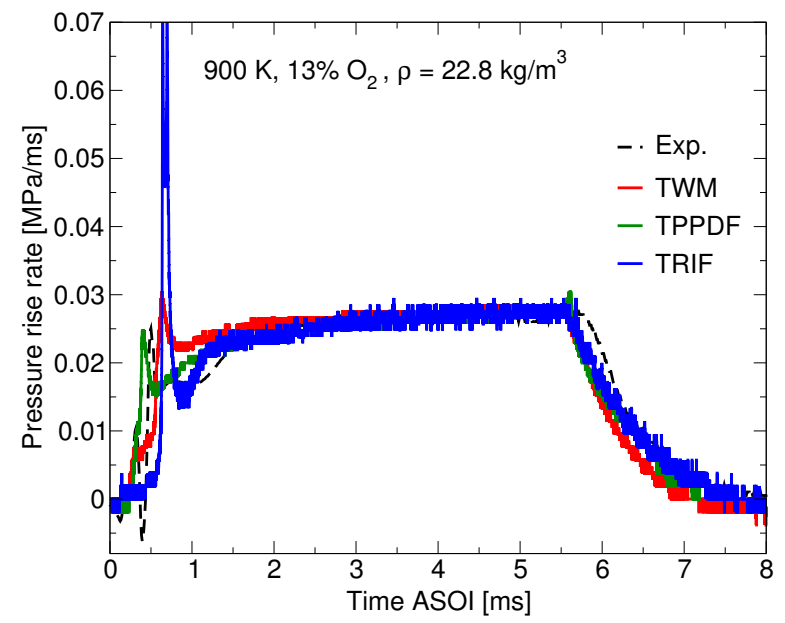

Figure 22: Comparison between experimental and computed pressure rise rate $(d p / d t)$ using the TWM, TPPDF and TRIF models at $900 \mathrm{~K}, 13 \% \mathrm{O}_{2}$

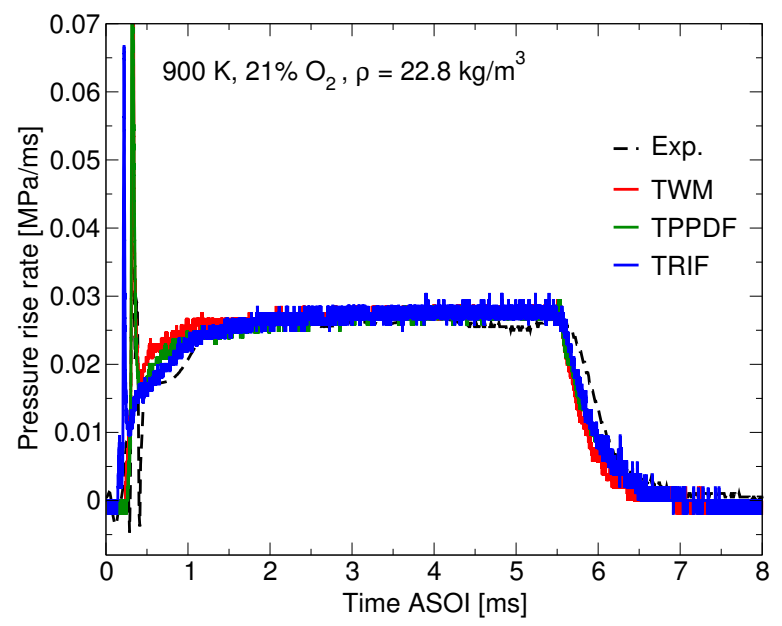

Figure 23: Comparison between experimental and computed pressure rise rate $(d p / d t)$ using the TWM, TPPDF and TRIF models at $900 \mathrm{~K}, 21 \% \mathrm{O}_{2}$

and temperatures. First, but not reported here, it was verified that all the tested approaches are able to follow the expected trends in terms of total amount of soot mass inside the domain. Soot grows when reducing the ambient oxygen concentration and increasing the ambient temperature. Maps of soot were acquired in a visible region of interest (ROI) with a $6.6 \times 67.2 \mathrm{~mm}$ extensions in the radial and axial direction, respectively. To predict the correct amount of soot mass in the ROI, the flame structure has to be properly described in terms of temperature, oxidizer and soot precursor distribution. Figs. 24(a)-(c) report a comparison between computed and experimental data of soot mass in the ROI for three different amount of ambient oxygen concentrations. The same soot tuning parameters were used for all the tested combustion models, showing a correct estimation of the soot formation onset in the ROI but an overprediction of the reached steady-state value which is significantly affected by the assumed flame structure. In particular the TRIF model seems to be the only one which is able to re- produce the experimental trend. In a future work, the sensitivity of the tested combustion approaches to soot model coefficients will be assessed together with a more detailed validation with optical data.
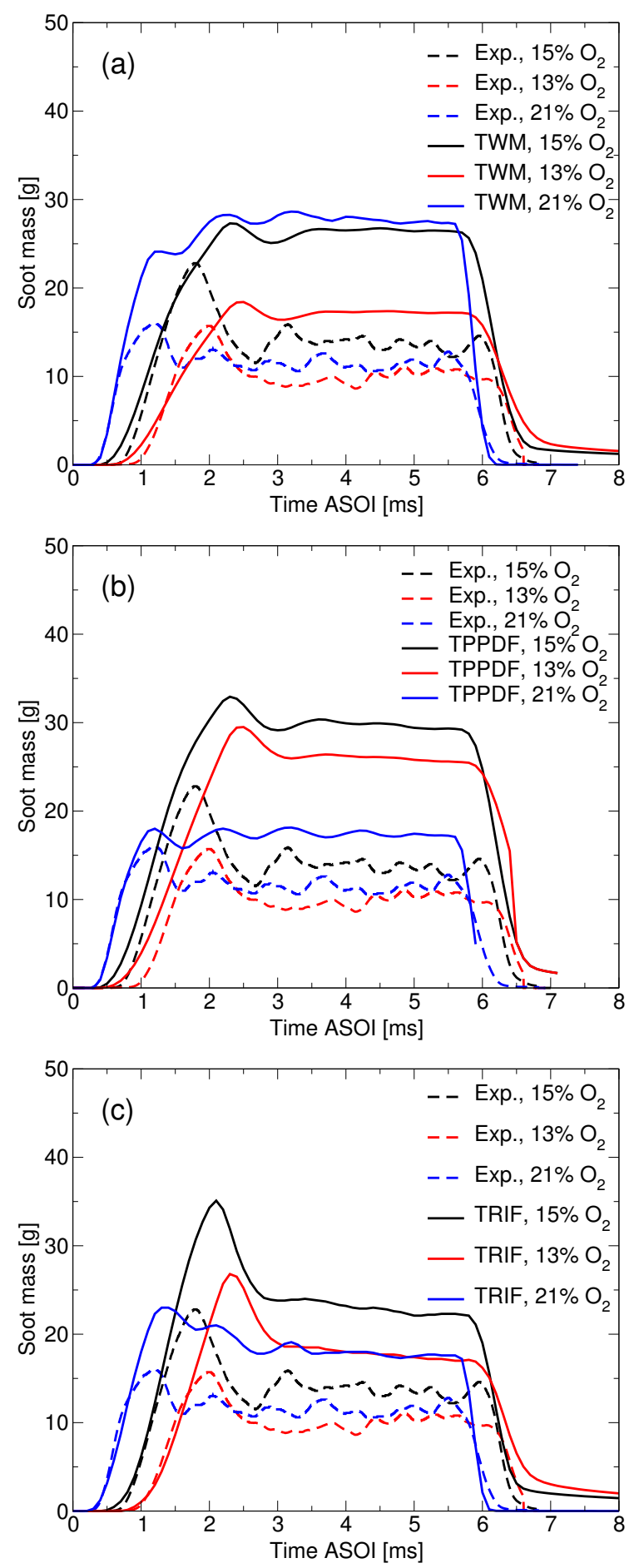

Figure 24: Comparison between experimental and computed data of soot mass: (a) TWM model; (b) TPPDF model; (c) TRIF model

So far, tabulated kinetics has shown a very good performance in terms of computational time and produces results which are consistent with the ones obtained with direct integration. All three tested models are able to repro- 
duce with a good level of accuracy ignition delay and liftoff trends. Concerning soot emissions, TRIF results show a good agreement with experimental data, while more investigations are required for the TWM and TPPDF models. Assessment and validation of combustion models with a detailed set of well-documented experiments at constantvolume conditions is a fundamental pre-requisite for successful engine simulations which will be discussed in the next session.

\section{Medium Duty Diesel Engine}

A Medium Duty Diesel (MDD) engine was simulated under three different operating conditions to validate the proposed combustion models. Details of the piston bowl geometry are provided in Fig. 25(b) together with the main engine data reported in Tab. 3.

\begin{tabular}{|c|c|}
\hline Bore & $96 \mathrm{~mm}$ \\
\hline Stroke & $104 \mathrm{~mm}$ \\
\hline Compression ratio & 18 \\
\hline IVC & $-145 \mathrm{deg}$ \\
\hline EVO & $110 \mathrm{deg}$ \\
\hline Swirl ratio & 1.3 \\
\hline Number of injector holes & 8 \\
\hline Nozzle hole diameter & $140 \mu \mathrm{m}$ \\
\hline
\end{tabular}

Table 3: Main geometry data of the MDD engine simulated in this work.

The spray-oriented grid was automatically generated using the algorithms presented in [25] and mesh details are provided in Fig. 25. Simulations start at IVC imposing a wheel-flow velocity profile whose intensity is proportional to the swirl number which was measured at the flow bench under steady-state flow conditions. The mesh has 80000 cells at IVC which are reduced to 15000 at TDC because during mesh motion the dynamic layering technique was employed [25]. The mesh resolution in the radial direction considers only 13 cells and is relatively coarse.

Three different operating points, representative of relevant speed and loads, were considered and their main details are reported in Tab. 4. It is possible to see that the selected conditions include variation of engine speed, load and EGR. When the engine runs at partial load, three injections are used (pre, pilot and main), while fuel is delivered only in a single injection at $100 \%$ load. Initial thermodynamic conditions at IVC were taken from 1D simulations of the whole engine system.

Simulations were run with three different chemistry tables, each one considering the corresponding level of oxygen which was found at IVC for the selected operating conditions. Residual gases were considered to be only $\mathrm{N}_{2}$. The tables were generated considering the same equivalence ratio values used for constant-volume combustion simula-
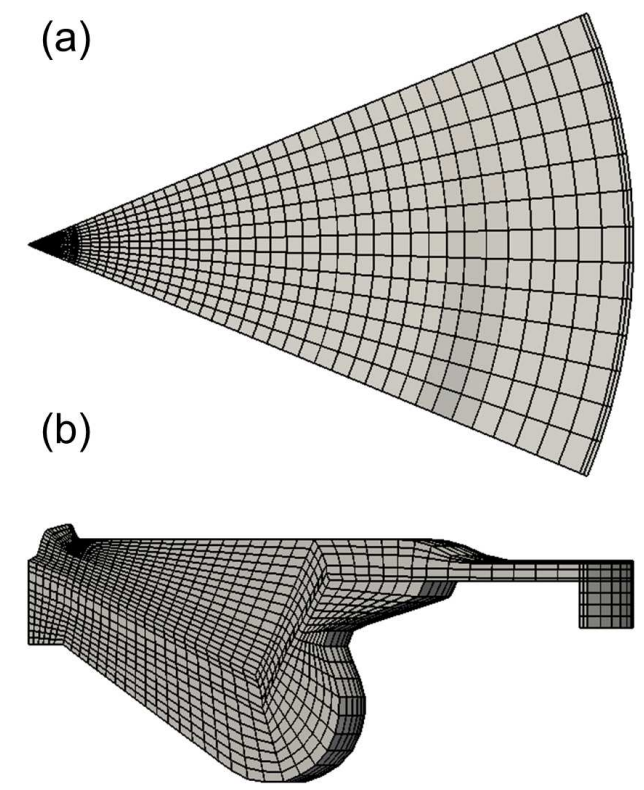

Figure 25: Computational mesh of the MDD engine: (a) Top view; (b) side view.

\begin{tabular}{|c|c|c|c|}
\hline & A25 & A75 & C100 \\
\hline Speed [rpm] & 2000 & 2000 & 3500 \\
\hline bmep [bar] & 4.5 & 13.5 & 14.5 \\
\hline No. of injections & 3 & 3 & 1 \\
\hline EGR [\%] & 22 & 13 & 0 \\
\hline Relative air/fuel ratio $\lambda$ & 1.8 & 1.1 & 1.4 \\
\hline
\end{tabular}

Table 4: Simulated operating conditions for the MDD Engine.

tions and including fuel evaporation effects on the initial reactor gas temperature. Large ranges of temperature and pressures were considered and the corresponding intervals are reported in Tab. 5. Towards the end of the expansion stroke it might happen that cylinder pressures and unburned temperatures $T_{u}$ become slightly lower than the lowest tabulated ones which are used to estimate the cylinder composition. Surely this introduces small errors and can affect the estimated $\mathrm{CO}$ emissions. However, predictions can be improved with a better pressure discretization, also including low pressure values.

\begin{tabular}{cc}
\hline Temperature $[\mathrm{K}]$ & $700,725,750,775$ \\
& $800,825,850,875$ \\
$900,925,950,975$ \\
$1000,1050,1100$ \\
12001300 \\
\hline Pressure [bar] & $20,40,60,80$ \\
& $100,120,140,160$ \\
$180,200,220,250$ \\
\hline
\end{tabular}

Table 5: Chemistry table discretization used for the simulation of the Medium Duty Diesel Engine

Figs. 26(a)-(c) report a comparison between computed and experimental data of in-cylinder pressure and heat release rate profiles for the three proposed combustion models. At 
least on this coarse grid, the performance of the three tested models is very similar, showing in general an overestimation of in-cylinder pressure. TRIF model has the best agreement with experimental data in the A25 case.
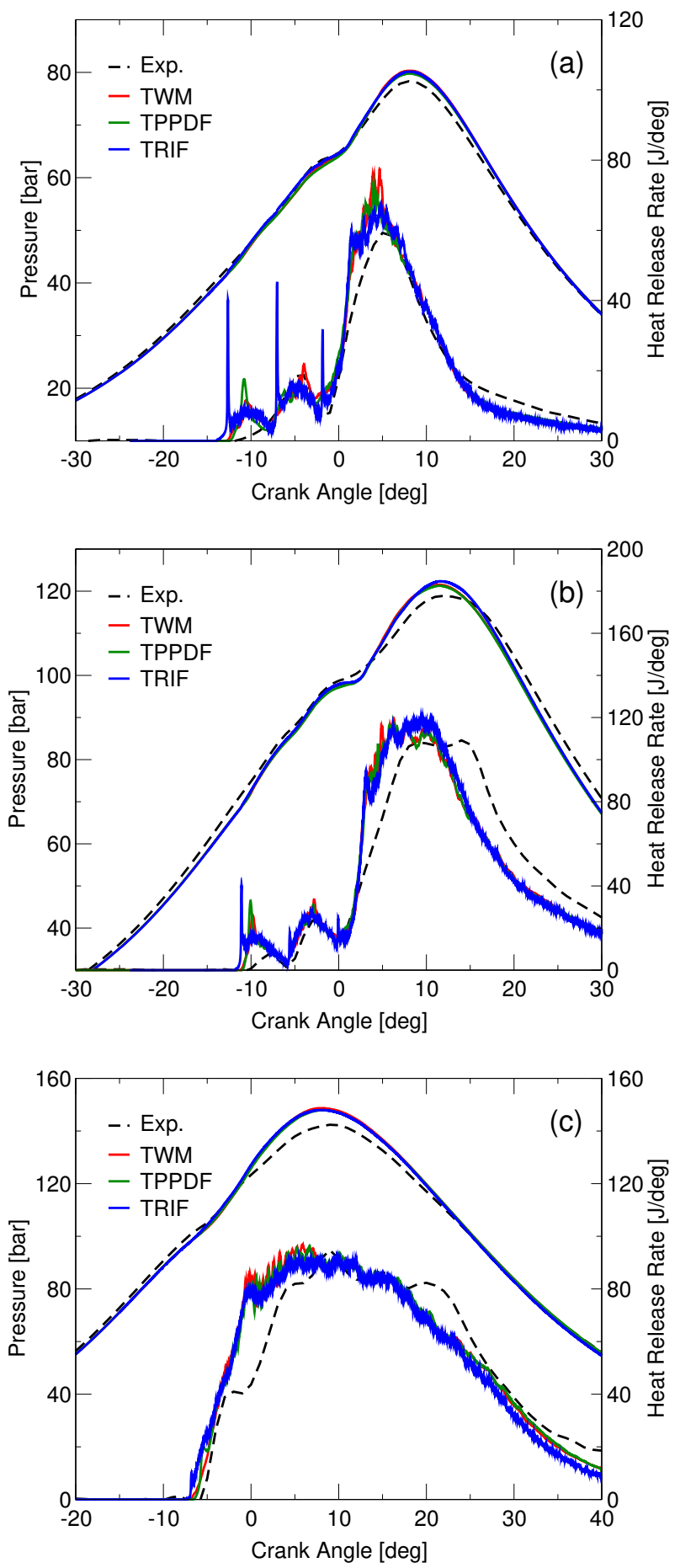

Figure 26: Comparison between experimental and computed data of in-cylinder pressure and heat release rate profiles using TWM, TPPDF and TRIF combustion models: (a) Operating A25; (b) Operating point A75; (c) Operating point C100.

In Figs. 27(a)-(b), computed and experimental data of NO emissions are shown. All the tested models correctly de- scribe the dependency of $N O_{x}$ emissions as function of maximum cylinder pressure and EGR rate. In terms of predictive capability, Fig. 27(b) shows that the best agreement is achieved when using the TWM model. However, it is necessary to carry out a mesh sensitivity analysis to better understand which is the best model: formation of $N O_{x}$ is strongly related to the distribution of mixture fraction and the use of a relatively coarse grid in the radial direction might affect the computed results.
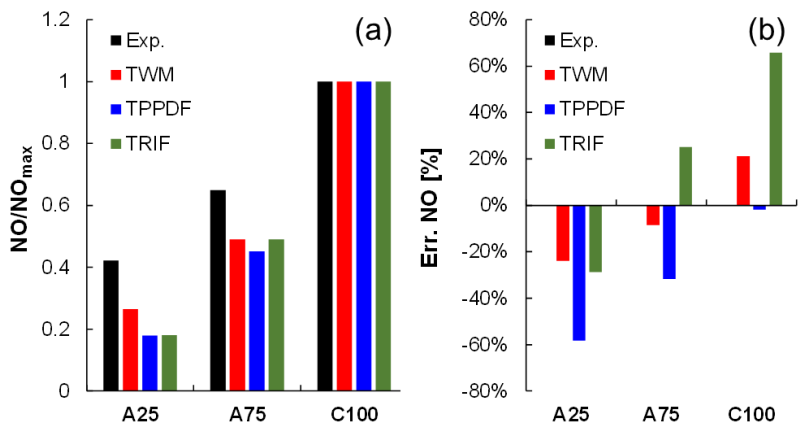

Figure 27: (a) Comparison between experimental and computed normalized $\mathrm{NO}_{x}$ emissions for the three selected operating points; (b) Computed relative error in $\mathrm{NO}_{x}$ estimation from TWM, TPPDF and TRIF models.

\section{Conclusions}

This work was focused on the development of combustion models based on tabulated kinetics for the simulation of Diesel engines. The chemistry table is based on homogeneous constant pressure reactor calculations and tabulated reaction rate values were used as source terms in progress variable transport equation by the proposed approaches which are based on three different flame structures: well-mixed, presumed PDF and representative interactive flamelets. To minimize the table size, local chemical composition is computed by means of the virtual species approach, with seven chemical species representing the full set of the detailed mechanism and preserving their main thermodynamic properties. Constant-volume combustion experiments were first used for the assessment and validation of the different combustion models. This made possible to verify their computational efficiency, showing speedup factors in the 5-20 range compared to the corresponding approaches where direct-integration was used. Consistency between tabulated kinetics and direct-integration was verified in terms of computed values of ignition delay, flame lift-off length, and heat release rate. The agreement with experimental data can be considered satisfactory, despite the fact that further validations are necessary for what concerns the prediction of soot emissions. Best results can be achieved either with TRIF or TPPDF models with the last one also able to predict the stabilization of the flame. Preliminary engine simulation results further confirm what was found for the spray-A case. In particular, turbulencechemistry interaction is necessary to correctly estimate in- 
cylinder pressure. Concerning $N O_{x}$ emissions, the trends are correctly captured but there is probably more work to do not only in the improvement of the mesh resolution, but also in the definition of a sub-model model which is more consistent with the flame structure assumptions made by TPPDF and TRIF. Tabulation of $N O_{x}$ formation rates, including also prompt mechanism into TWM and TPPDF models can probably improve the results presented in this work also taking turbulence-chemistry interaction into account.

\section{Contact Information}

Prof. Tommaso Lucchini

Department of Energy, Politecnico di Milano

Via Lambruschini, 4

20156 Milano, Italy

tommaso.lucchini@polimi.it

\section{References}

1. Y. Funayama, H. Nakajima, and K. Shimokawa. A Study on the Effects of a Higher Compression Ratio in the Combustion Chamber on the Diesel Engine Performance. SAE Paper, 2016-01-0722, 2016.

2. M. Hashimoto, Y. Aoyagi, M. Kobayashi, T. Murayama, Y. Goto, and $\mathrm{H}$. Suzuki. BSFC Improvement and NOx Reduction by Sequential Turbo System in a Heavy Duty Diesel Engine . SAE Paper, 2012-010712, 2012.

3. P. F. Flynn, R. P. Durret, G. L. Hunter, A. O. zur Loye, O. C. Akinyemi, J. E. Dec, and C. K. Westbrook. Diesel Combustion: An Integrated View Combining Laser Diagnostics, Chemical Kinetics and Empirical Validation. SAE Paper, 1999-01-0509, 1999.

4. G. Bruneaux. Mixing Process in High Pressure Diesel Jets by Normalized Laser Induced Exciplex Fluorescence - Part I: Free Jet. SAE Paper, 2005-01-2100, 2005.

5. G. D'Errico, T. Lucchini, F. Contino, M. Jangi, and X.S. Bai. Comparison of well-mixed and multiple representative interactive flamelet approaches for diesel spray combustion modelling. Combustion Theory and Modelling, 18(1):65-88, 2014.

6. Y. Pei, E. R. Hawkes, and S. Kook. Transported probability density function modelling of the vapour phase of an n-heptane jet at diesel engine conditions. Proceedings of the Combustion Institute, 34(2):3039 3047, 2013.

7. H. Barths, C. Hasse, and N. Peters. Computational fluid dynamics modelling of non-premixed combus- tion in direct injection diesel engines. International Journal of Engine Research, 1 (3):pp. 249-267, 2000.

8. Y. M. Wright, K. Boulouchos, G. De Paola, and E. Mastorakos. Multi-dimensional Conditional Moment Closure Modelling Applied to a Heavy-duty Common-rail Diesel Engine. SAE Paper, 2009-010717, 2009.

9. R. Diwakar and S. Singh. NOx and Soot Reduction in Diesel Engine PCCI Combustion: A Computational Investigation. International Journal of Engine Research, 9(3):pp. 195-214, 2008.

10. M. Sjoberg and J. E. Dec. Smoothing HCCI heatrelease rates using partial fuel stratification with twostage ignition fuels. SAE Paper, 2006-01-0629, 2006.

11. F. Contino, H. Jeanmart, T. Lucchini, and G. DErrico. Coupling of in situ adaptive tabulation and dynamic adaptive chemistry: An effective method for solving combustion in engine simulations. Proceedings of the Combustion Institute, Vol. 33(2):pp. 3057-3064, 2011.

12. M. Embouazza, D. C. Haworth, and N. Darabiha. Implementation of Detailed Chemical Mechanisms Into Multidimensional Cfd Using in Situ Adaptive Tabulation: Application to HCCI Engines. SAE Paper, 200201-2773, 2002.

13. A. Babajimopoulos, D. N. Assanis, D. L. Flowers, S. M. Aceves, and R. P. Hessel. A fully coupled computational fluid dynamics and multi-zone model with detailed chemical kinetics for the simulation of premixed charge compression ignition engines. International Journal of Engine Research, Vol. 6:497-512, 2005.

14. M. Raju, M. Wang, M. Dai, W. Piggott, and D. Flowers. Acceleration of Detailed Chemical Kinetics Using Multi-zone Modeling for CFD in Internal Combustion Engine Simulations. SAE Paper, 2012-01-0135, 2012.

15. T. Lucchini, G. D’Errico, F. Contino, and M. Jangi. Towards the use of eulerian field pdf methods for combustion modeling in ic engines. SAE International Journal of Engines, 7(1):286-296, 2014.

16. U. Maas and S. B. Pope. Simplifying Chemical Kinetics: Intrinsic Low-Dimensional Manifolds in Composition Space. Combustion and Flame, Vol. 88:239264, 1992.

17. M.J. Wankhede, F.A. Tap, P. Schapotschnikow, and W.J.S. Ramaekers. Numerical study of unsteady flowfield and flame dynamics in a gas turbine model combustor. Proceedings of the ASME Turbo Expo, 4A, 2014. 
18. C. Pera, O. Colin, and S. Jay. Development of a fpi detailed chemistry tabulation methodology for internal combustion engines [dveloppement d'une tabulation fpi pour la prise en compte de la chimie complexe dans la simulation 3d moteurs]. Oil and Gas Science and Technology, 64(3), 2009.

19. C. Bekdemir, L.M.T. Somers, and L.P.H. de Goey. Modeling diesel engine combustion using pressure dependent flamelet generated manifolds. Proceedings of the Combustion Institute, 33(2):2887 - 2894, 2011.

20. H. Lehtiniemi, Y. Zhang, R. Rawat, and F. Mauss. Efficient 3-D CFD Combustion Modeling with Transient Flamelet Models. SAE Paper, 2008-01-0957, 2008.

21. B. Naud, R. Novella, J.M. Pastor, and J.F. Winklinger. Rans modelling of a lifted $\mathrm{h} 2 / \mathrm{n} 2$ flame using an unsteady flamelet progress variable approach with presumed pdf. Combustion and Flame, 162(4):893-906, 2015.

22. J-B. Michel, O. Colin, C. Angelberger, and D. Veynante. Using the tabulated diffusion flamelet model adf-pcm to simulate a lifted methaneair jet flame. Combustion and Flame, 156(7):1318 - 1331, 2009.

23. J-B. Michel, O. Colin, C. Angelberger, and D. Veynante. A tabulated diffusion flame model applied to diesel engine simulations. International Journal of Engine Research, 15:346 - 369, 2009. 2013.

24. G. D'Errico, T. Lucchini, A. Onorati, and G.Hardy. Computational fluid dynamics modeling of combustion in heavy-duty diesel engines. International Journal of Engine Research, 16 (1):pp. 112-124, 2015.

25. T. Lucchini, A. Della Torre, G. D'Errico, G. Montenegro, M. Fiocco, and A. Maghbouli. Automatic Mesh Generation for CFD Simulations of Direct-Injection Engines. SAE Paper, 2015-01-0376, 2015.

26. M. Ihme, L. Shunn, and J. Zhang. Regularization of reaction progress variable for application to flameletbased combustion models. Journal of Computational Physics, 231(23):7715-7721, 2012.

27. R. Aglave, U. Riedel, and J. Warnatz. TurbulenceChemistry Interactions in CFD Modelling of Diesel Engines. Combustion Theory and Modelling, 12(2):pp. 305-328, 2008.

28. A. Krisman, J.C.K. Tang, E. R. Hawkes, D. O. Lignell, and J. H. Chen. A $\{$ DNS $\}$ evaluation of mixing models for transported $\{\mathrm{PDF}\}$ modelling of turbulent nonpremixed flames. Combustion and Flame, 161(8):2085 $-2106,2014$.

29. J. B. Heywood. Internal Combustion Engine Fundamentals. McGraw-Hill, 1988.
30. K.M. Leung, R.P. Lindstedt, and W.P. Jones. A simplified reaction mechanism for soot formation in nonpremixed flames. Combustion and Flame., 87:289305, 1991.

31. M. Bolla, D. Farrace, Y. M. Wright, K. Boulouchos, and E. Mastorakos. Influence of turbulencechemistry interaction for $n$-heptane spray combustion under diesel engine conditions with emphasis on soot formation and oxidation. Combustion Theory and Modelling, 18(2):330-360, 2014.

32. T. Kim and Y. Kim. Interactive transient flamelet modeling for soot formation and oxidation processes in laminar non-premixed jet flames. Combustion and Flame., 162:1660-1678, 2015.

33. R. D. Reitz. Modeling Atomization Processes In High Pressure Vaporizing Sprays. Atomization and Spray Technology, Vol. 3:pp. 309-337, 1987.

34. A. Frassoldati, G. D’Errico, T. Lucchini, A. Stagni, A. Cuoci, T. Faravelli, A. Onorati, and E. Ranzi. Reduced kinetic mechanisms of diesel fuel surrogate for engine $\{\mathrm{CFD}\}$ simulations. Combustion and Flame, 162(10):3991 - 4007, 2015.

35. M. Meijer, B. Somers, J. Johnson, J. Naber, S.-Y. Lee, L. M. Malbec, G. Bruneaux, L. M. Pickett, M. Bardi, R. Payri, and T. Bazyn. Engine combustion network (ecn): Characterization and comparison of boundary conditions for different combustion vessels. Atomization and Sprays, 22(9):777-806, 2012.

36. J. Manin, L. Pickett, and S. Skeen. Two-Color Diffused Back-Illumination Imaging as a Diagnostic for TimeResolved Soot Measurements in Reacting Sprays. SAE International Journal of Engines, 6(1):1908-1921, 2013.

37. L. M. Pickett, C. L. Genzale, G. Bruneaux, L.M L.-M. Malbec, L. Hermant, C. Christiansen, and J. Schramm. Comparison of diesel spray combustion in different high-temperature, high-pressure facilities. SAE International Journal of Engines, 3(2):156-181, 2010.

38. L. M. Pickett, S. Kook, and T. Williams. Visualization of diesel spray penetration, cool-flame, ignition, high-temperature combustion, and soot formation using high-speed imaging. SAE International Journal of Engines, 2(1):439-459, 2009. 